\title{
The role of event rate, temporal expectancy, and sensory modality in continuous performance of children and adults
}

\author{
Lori M. Curtindale ${ }^{1}$ \\ Published online: 15 September 2020 \\ (C) The Psychonomic Society, Inc. 2020
}

\begin{abstract}
This study explored developmental differences in the effects of event rate, temporal expectancy, and sensory modality on continuous performance. Children (ages 7-8 years) and college-aged adults completed visual and auditory continuous performance tasks (CPTs) that were equated at an intermediate $\left(20\right.$ events/min) rate using the perceptual sensitivity index $\left(d^{\prime}\right)$ and then were compared at faster ( 40 events $/ \mathrm{min})$ and slower $(10$ events $/ \mathrm{min})$ rates to determine the influence of event rate on continuous performance of children and adults. To investigate the effects of temporal expectancy, $20 \%$ of the critical signals and neutral events occurred early or late relative to the regular rhythm of the task. The findings (a) suggest that event rate influences continuous performance differently for children and adults, (b) highlight the role of temporal expectancy in continuous performance, and (c) reveal differences in the effects of event rate and temporal expectancy on visual and auditory continuous performance.
\end{abstract}

Keywords Attention $\cdot$ Temporal processing $\cdot$ Development

\section{Introduction}

Continuous performance tasks (CPTs) are commonly used to assess attention of children and adults in clinical and research settings (Aylward, Brager, \& Harper, 2002; Chee, Logan, Schachar, Lindsay, \& Wachsmuth, 1989; Roebuck, Freigang, \& Barry, 2016; van der Meere \& Stemerdink, 1999). In CPTs participants attend to a temporally extended stream of events, responding to critical signals and withholding responses to neutral events. Although the basic design is the same, CPTs use a variety of task parameters (e.g., visual vs. auditory modality) making it difficult to compare across studies and generalize results. Many factors found to affect adult performance have been overlooked in studies of typically developing children (Laurie-Rose, Bennett-Murphy, Curtindale, Granger, \& Walker, 2005; Laurie-Rose, Pempek, $\&$ Curtindale, 2015). For example, temporal structure variation (e.g., event rate), studied extensively in adults, impacts performance of children with attention deficit hyperactivity disorder (ADHD) on a variety of tasks (Metin, Roeyers,

Lori M. Curtindale

curtindale@ lycoming.edu

1 Department of Psychology, Lycoming College, One College Place, Campus Box 1, Williamsport, PA 17701, USA
Wiersema, van der Meere, \& Sonuga-Barke, 2012); however, less is known about its effects on typically developing attention. The goal of the present study was to fill a gap in the literature by comparing the effects of variations in event time structure - event rate and temporal expectancy - on performance of children (ages 7-8 years) and adults during visual and auditory CPTs.

\section{Effects of temporal structure variation}

Events in a CPT unfold over time, providing information about the temporal structure of the task. Dynamic attending theory (Jones \& Boltz, 1989) and associated entrainment models (McAuley, Jones, Holub, Johnston, \& Miller, 2006) provide a framework for understanding the influence of temporal structure on event processing - detection and discrimination - in a variety of settings. For example, there is evidence that people use temporal structure to develop predictions and anticipate future visual and auditory events, and that these predictions are more accurate when events occur at temporally expected compared to unexpected points in time (Jones, Moynihan, MacKenzie, \& Puente, 2002; Miller, Carlson, \& McAuley, 2013). Although temporal properties of events guide attention across the life span, there is evidence of developmental differences in preferred event rate and event tracking (McAuley et al., 2006). For example, children demonstrate a 
preference for fast compared to slow rates when listening to and tapping sequences that slows with age. Temporal structure and its variations (i.e., event rate and temporal expectancy) are important factors to consider when comparing CPT performance of children and adults.

\section{Event rate}

Event rate, defined by the number of events presented in a task per minute, is one of the most important factors to affect adult attention (Parasuraman \& Davies, 1977). Early research using visual and auditory tasks with adults has reported superior performance (i.e., hits/correct detections and perceptual sensitivity) when events were presented at a slow compared to a fast event rate (Davies \& Parasuraman, 1982; Jerison \& Pickett, 1964; Parasuraman, 1979; Warm \& Jerison, 1984). Recently, Singh and colleagues found the same inverse relationship between visual performance and event rate (Tiwari, Singh, \& Singh, 2009; Yadav, Dubey, \& Singh, 2016). However, studies with a non-clinical sample of both children and adults, in the visual domain, report better performance (i.e., perceptual sensitivity) when events are presented at a fast compared to a slow event rate (Laurie-Rose et al., 2005; Laurie-Rose, Bennett-Murphy, Schickedantz, \& Tucci, 2001). Inconsistent findings on the effects of event rate may be the result of performance measures and task parameters that have co-varied within and between studies. Relevant in the present study are the task parameters of signal probability (frequency), task duration, and rate classification (i.e., specific rates used for fast and slow conditions).

Typically, signal probability is defined as the ratio of critical signals, which require a response, to the total number of events in a task (See, Howe, Warm, \& Dember, 1995). Varying levels of signal probability make it difficult to compare studies of event rate and generalize results. When probability is high compared to low, children and adults are less conservative, correctly identifying more critical signals and committing more false alarms, and they respond more quickly (Baddeley \& Colquhoun, 1969; Jenkins, 1958; Laurie-Rose et al., 2001; Warm \& Alluisi, 1971). Jerison and Pickett's (1964) investigation of event rate held the number of critical signals constant for both the fast and slow event rates, resulting in higher probability of a critical signal in the slow than in the fast event-rate conditions. Thus, the reported differences between the fast and slow event rates may be a result of shifts in response bias rather than the effects of event rate (Davies \& Parasuraman, 1982). To address this limitation, signal probability in the present study was held constant across all event-rate conditions.

Another factor that may account for the differences in the effects of event rate on continuous performance is task duration. Studies of event rate have employed a wide range of task durations - 10-80 min (Jerison \& Pickett, 1964; van der
Meere \& Stemerdink, 1999). It is critical to note these differences in task duration, because as time on task increases performance can decline. In addition, when signal probably is held constant across event rates, participants experience more critical signals in fast compared to slow tasks; therefore, performance may decline more quickly when event rate is fast compared to slow. The combined effects of signal probability and task duration should be considered when examining overall performance and when directly comparing performance of children and adults. In the present study, task duration was held constant - 10 min of CPT performance at all event rates was analyzed for both children and adults. ${ }^{1}$

Different classifications of fast and slow event rates may also account for conflicting results. Although the boundary between slow and fast event rates has been identified as 24 events/min in the adult literature, this is the only guideline for choosing values for an event rate task (Parasuraman \& Davies, 1977). Research manipulating event rate has used slow rates ranging from 5 to 15 events/min and fast rates ranging from 30 to 60 events $/ \mathrm{min}$. This variability and the use of only two rates makes it difficult to determine whether the effects are driven by the fast or slow rate. For example, an inverse relationship between performance and event rate may be due to superior performance at the slow event rate related to a preference for a slower rate, poor overall performance at the fast event rate, or a combination of both. Several researchers, using clinical samples of children, have introduced an intermediate rate that is not too fast or too slow. These studies report superior performance (i.e., overall hits and false alarms) at an intermediate rate (ranging from 10 to 30 events/min), compared to fast or slow event rates (Chee et al., 1989; van der Meere \& Stemerdink, 1999). The present study incorporated an intermediate event rate, in addition to the conventional fast and slow event rates; all were classified using the 24 events/min boundary and within the range of rates employed in previous research.

The focus of CPTs is often on overall performance, especially when task duration is short (e.g., Roebuck et al., 2016); however, performance can also be measured through the decline in performance (vigilance decrement) that can occur when participants sustain their attention for an extended period (Ballard, 1996). Researchers report a variety of CPT performance measures: hits (correct detections), false alarms (errors of commission), misses (errors of omission), reaction times to hits (RTs), and signal detection indices (Riccio, Reynolds, Lowe, \& Moore, 2002). Signal detection indices provide separate estimates of perceptual ability (perceptual sensitivity, $d^{\prime}$ ) and willingness to make a response (response

\footnotetext{
${ }^{1}$ Adults completed a 20-min CPT, but only data for the first 10 min were included in the analysis. When data were analyzed using the entire $20 \mathrm{~min}$, the pattern of results was similar. Notable differences include a significant threeway interaction between age, modality, and event rate for $d$ ' and a significant two-way interaction between modality and temporal expectancy for $\mathrm{c}$.
} 
bias, c), which may not be directly related to the proportion of hits and false alarms (Green \& Swets, 1966; Macmillan \& Creelman, 1991; See et al., 1995). Differences in measures reported across studies may contribute to inconsistent findings on the effects of event rate on CPT performance. The present study used $d$, , c, and RT to assess overall performance on visual and auditory CPTs. Although hits and false alarms are not independent of $d^{\prime}$ and $\mathrm{c}$, they were included in the analyses of event rate effects to allow for comparisons to previous studies that only reported hits and/or false alarms.

\section{Temporal expectancy}

Another variation in temporal structure that influences CPT performance is temporal expectancy. Typically, events in a CPT appear with temporal regularity, at expected points in time (Davies \& Parasuraman, 1982). This temporal certainty (expectancy) allows participants to predict when the next event will occur and take "time-outs" in observing when an event is not being presented. However, in temporally uncertain tasks, participants cannot predict when the next event will occur; they must constantly monitor the display, and performance suffers. Previous research has demonstrated superior performance when critical signals are presented with temporal regularity compared to tasks with unpredictable critical signals, a result known as the signal regularity effect (Adams \& Boulter, 1964; Helton et al., 2005; Scerbo, Warm, Doettling, Parasuraman, \& Fisk, 1987; Warm, Dember, Murphy, \& Dittmar, 1992). Other studies report similar enhancements in performance when background (neutral) events are presented with temporal regularity (synchrony) compared with when they are irregular (asynchronous), a finding termed the event asynchrony effect (Richter, Senter, \& Warm, 1981; Scerbo et al., 1987; Scerbo, Warm, \& Fisk, 1986).

The signal regularity and event asynchrony effects reported in studies that employ CPTs are consistent with the dynamic attending literature. Jones and colleagues (Jones \& Boltz, 1989; Jones et al., 2002; Penel \& Jones, 2005) investigated the effects of specific timing manipulations, such as rhythmic expectancy, on attention. In these tasks, participants used future-oriented attending to develop temporal predictions and anticipate the occurrence of the next event following a temporally regular sequence. While much of this research has utilized sequences to entrain attention prior to presenting a target event, dynamic attending theory has also been applied to tasks with target events embedded within a context sequence. For example, in an auditory oddball paradigm, participant judgments about the duration of oddball events were most accurate when the oddball was temporally expected (on time) compared to oddballs that were unexpected (early or late) relative to the context sequence in which the oddballs were embedded (McAuley \& Fromboluti, 2014). These studies highlight the advantage of predictable events rather than events that occur unexpectedly; however, when participants have temporal expectancies, they may also notice events that are early or late compared to the rhythm of the task (Penel $\&$ Jones, 2005). This reactive attending occurs when individuals shift their attention toward unexpected events after they have occurred. Enhancement of unexpected events compared to on-time events is known as a capture effect (Penel \& Jones, 2005). The present study sought to explore the effects of temporal expectancy on continuous performance by presenting a limited number of temporally unexpected probe events that occurred early or late relative to the expected timing of the event stream rather than manipulating the timing of the entire task.

\section{Effects of sensory modality}

Sensory modality has been identified as a significant variable in the adult literature on attention (Davies \& Parasuraman, 1982). In addition, there is an increased interest in understanding the relationship between visual and auditory CPTs as they relate to ADHD because auditory tasks may be more closely related to performance in the classroom than visual tasks (Lasee \& Choi, 2013; Schmidt, Simões, \& Novais Carvalho, 2016). Although investigations on differences between visual and auditory tasks exist, less attention has been given to the comparability of the tasks. There is an inconsistent relationship between performance in visual and auditory tasks that is likely due to variations in task demands, which is influenced by task parameters (e.g., event rate, signal probability) and the degree of coupling between the observer and the display (Curtindale, Laurie-Rose, Bennett-Murphy, \& Hull, 2007). Visual tasks are considered loosely coupled, whereas auditory tasks are tightly coupled. In visual tasks, participants can move their eyes and heads away from the display, which may cause them to miss the presentation of some stimuli (Hatfield \& Loeb, 1968). Auditory tasks are often presented through headphones, leading participants to be "tied in" to the stimulation, making it more difficult for stimuli to be missed (Hatfield \& Soderquist, 1970; Loeb \& Binford, 1971). The early adult literature suggests that without establishing comparable tasks, the auditory modality yields superior performance (i.e., hits and reaction time) compared to visual tasks (cf., Warm \& Jerison, 1984). Studies designed to control for equivalency produced inconsistent results - comparable performance for the visual and auditory tasks (See et al., 1995) and better performance (i.e., hits) in the visual than the auditory task for children and adults (Curtindale et al., 2007). Clearly, more research is necessary. A goal of the present study was to determine the effects of other variables, such as age, event rate, and temporal expectancy on visual and auditory CPT performance. To address the issue of task demands, 
visual and auditory tasks of comparable difficulty were developed for children and adults at an intermediate event rate.

\section{The current study}

Overall, questions about the effects of event rate and temporal expectancy/uncertainty on visual and auditory continuous performance remain unanswered. First, further research is necessary to determine if a fast, intermediate, or slow event rate yields superior performance and if the effects of event rate differ for children and adults. Results of previous studies examining event rate are conflicting. There is some support for superior performance with slow event rates, while other studies suggest an advantage with fast event rates. These inconsistent results may be due to differences in the task parameters. No previous studies of event rate have controlled task parameters when directly comparing continuous performance of children and adults. Of interest in this paper is whether there are modality differences in the effects of event rate on performance and whether these differences are influenced by age. One additional possibility that has not been previously considered is that effects of event rate may be different for visual and auditory tasks.

Although previous studies report poorer performance for temporally irregular tasks, the effects of temporally unexpected probes, including critical signals and neutral events, have not been explored in a CPT. Research examining effects of rhythmic temporal expectancy in pitch discrimination (Jones et al., 2002) and auditory oddball (McAuley \& Fromboluti, 2014) tasks suggest that temporally expected events are highlighted and therefore processed more accurately than unexpected events. In contrast, other studies have found temporally unexpected information to be more salient, as indicated by more responding to unexpected events (Penel \& Jones, 2005). These results suggest that rhythmic expectancy may play an important role in attending. Relevant to the present study was whether this work on rhythmic expectancy generalizes to a CPT and whether effects of rhythmic expectancy on attending are affected by age and modality. The present study investigated these research questions. First, a preliminary study was conducted to create equivalent visual and auditory CPTs for children and adults. Previous research investigating developmental differences in performance identified task equivalency as an important factor to consider when comparing performance of children and adults in the same study (Curtindale et al., 2007; Laurie-Rose et al., 2005). It is often challenging for children to successfully complete tasks designed for adults, while adults perform at ceiling levels in tasks designed for children. These performance differences provide insight into adults' superior perceptual abilities and attention skills. However, age differences found when exploring other factors, such as event rate, may be due to nonequivalent tasks rather than to the factor itself. To address concerns about the effects of task comparability on event rate, the preliminary study equated visual and auditory tasks for children and adults at an intermediate event rate. The main study explored the effects of event rate and temporal expectancy on visual and auditory CPT performance of children and adults. Participants completed both visual and auditory tasks presented at a fast, intermediate, or slow event rate. Three event rates were used to determine what happens when event presentation rate speeds up and slows down, and whether the effects are different for children and adults in visual and auditory tasks. In addition, a few unexpectedly early and late events were introduced to determine the effects of temporal expectancy.

\section{Preliminary study}

The aim of the preliminary study was to design comparable visual and auditory CPTs for children and adults at an intermediate event rate (20 events/min). Tasks were calibrated using the signal detection index perceptual sensitivity $d^{\prime}$, a measure of an observer's ability to detect changes in stimuli. This measure has been used to match tasks across age, between older and college-aged adults (Parasuraman, Nestor, \& Greenwood, 1989) and between children and collegeaged adults (Laurie-Rose et al., 2005), across modality (Hatfield \& Loeb, 1968), and across both age and modality (Curtindale et al., 2007). In the preliminary study, children and adults completed 10-min visual and auditory tasks with a variety of stimulus pairs to calibrate tasks to a $d^{\prime}$ value between 1.5 and 3.5 (with a target $d^{\prime}$ of 2.5), a range established as moderately difficult to moderately easy (Craig, 1984). For each task, scatterplots of all stimulus pairs (i.e., the difference between the critical signal and the neutral event) and $d$ ' scores were used to determine which stimulus pairs would yield average $d$ ' scores of 2.5. A final group of children and adults completed visual and auditory tasks with these stimulus pairs to evaluate whether the tasks were comparably difficult.

\section{Method}

\section{Participants}

Twenty-five elementary school children were recruited from communities in Northwest Ohio for the task matching process; they were paid $\$ 10$ for their participation. Parents were asked to report any diagnoses or history of attention problems. Data for 11 children were omitted because they did not fall in the study's target age range (ages 7-8 years). Eighty-four adults, undergraduate students at a university in Northwest Ohio, participated in the task-matching process for course credit. Participants were asked to report any diagnoses and/ 
or history of attention problems on a background questionnaire. Data for 14 participants were omitted from the final analyses for exclusionary criteria: diagnoses or concerns related to attention $(n=2)$, hearing screening failure $(n=1)$, and basal and ceiling performance $(n=11)$ (see Curtindale et al., 2007; Laurie-Rose et al., 2005).

Following the task-matching process, an additional 14 children and 19 adults were recruited to complete visual and auditory tasks with the matched stimulus pairs. Three adults were omitted for exclusionary criteria: diagnoses or concerns related to attention $(n=2)$, hearing screening failure $(n=1)$. The final sample consisted of 14 children, ranging in age from 7 years 7 months to 8 years 10 months, with an average of 8 years 3 months, and 16 adults, ranging in age from 18 to 27 years, with an average of 19 years 6 months.

\section{Procedure}

Participants completed a visual task, an auditory task, or both a visual and an auditory task on separate occasions at least $24 \mathrm{~h}$ apart. Prior to testing, all participants were read the task instructions, presented with examples of the stimuli, and completed a 2-min practice block, consisting of 40 events. Following the practice block, they experienced a continuous series of 200 test events (comprised of critical signals and neutral events) for $10 \mathrm{~min}$. Participants were asked to respond to the critical signal by pressing a designated button on a response box and withhold responses to the neutral event. Events lasted $100 \mathrm{~ms}$ and were presented at an intermediate rate (20 events/min). The probability of a critical signal was $30 \%$. All aspects of stimulus presentation and response collection for the visual and auditory tasks were controlled by the E-prime software package (Schneider, Eschman, \& Zuccolotto, 2002). In the visual task, participants were asked to discriminate between a small square designated the neutral event and the slightly larger square designated the critical signal. The squares were outlined by 1-mm black lines and presented on a white background. For both children and adults, the neutral event was held constant in size $1.25 \times$ 1.25 in. $(31.75 \times 31.75 \mathrm{~mm})$. In the auditory task, participants were asked to discriminate between a standard tone designated the neutral event and a tone with a slightly higher frequency designated the critical signal. All tones were generated on a Dell PC with a 32-bit Soundblaster Live soundcard and presented through headphones at a comfortable listening level $(\sim 70 \mathrm{~dB})$. For both children and adults, the neutral event was held constant at $440 \mathrm{~Hz}$.

The preliminary study began with an adaptive matching procedure for the adult visual and auditory tasks. Two participants completed the task with the same differences between the neutral event and critical stimulus (e.g., 5\%). If they produced an average $d$ ' score greater than 2.5, the stimulus difference was decreased by half (e.g., 2.5\%) for the next two participants, whereas if participants produced average $d$ ' scores less than 2.5, the stimulus difference was increased by half (e.g., 7.5\%). However, if the average $d^{\prime}$ 'was within 0.05 of 2.5 , four additional adults completed the task with the initial stimulus differences (e.g., 5\%). If the average $d$ ' for those six participants was not within 0.05 , the stimulus difference was adjusted by half again. This adaptive procedure narrowed the range of appropriate stimulus differences, but was not successful in identifying appropriate stimulus differences for the calibrated tasks. Therefore, additional data were collected with a variety of stimulus pairs (e.g., $3 \%, 6 \%$, and $12 \%$ ) and used in conjunction with adaptive matching data for a curve-fitting procedure. All stimulus differences assessed and corresponding $d^{\prime}$ 'scores were plotted and fit with linear equations. The equations were used to determine the stimulus difference (intercept) associated with a $d$ ' value of 2.5. Stimuli for children were matched with the same curve-fitting procedure, using adult matching data as a reference (see Table 1). A final group of children and adults completed visual and auditory tasks with the matched stimulus pairs (see Table 2) to determine if the tasks were equivalent.

\section{Results and discussion}

Perceptual sensitivity $\left(d^{\prime}\right)$ scores were calculated for the final group of children and adults. For the visual task, mean $d$ ' scores were $2.27(S D=.74)$ for children $(n=10)$ and 2.27 $(S D=.69)$ for adults $(n=9)$. Mean $d^{\prime}$ scores for the auditory task were $2.38(S D=1.14)$ for children $(n=12)$ and $2.39(S D$ $=1.09)$ for adults $(n=7)$. All values were slightly less than the target $d$ ' value of 2.5 ; however, of primary interest was whether the tasks were equivalent. A 2 (age) $\times 2$ (modality) univariate analysis of variance (ANOVA) showed no significant effects of age, $F(1,33)=0.00, p>.99, \eta_{\mathrm{p}}{ }^{2}<.001$, or modality, $F(1,33)=0.13, p=.73, \eta_{\mathrm{p}}{ }^{2}<.01$. There was also no significant interaction between age and modality, $F(1,33)=$ $0.00, p=.98, \eta_{\mathrm{p}}^{2}<.001$. The absence of a significant difference in traditional null hypothesis testing does not necessarily demonstrate statistical equivalence; therefore, the ANOVA was accompanied by equivalence testing.

One method commonly used to test for equivalence of means is the two one-sided test (TOST) procedure (Lakens, 2017; Schuirmann, 1987). This procedure tests whether an observed effect size differs from the smallest effect size of interest, defined by a lower and upper bound (Lakens, 2017). A significant result indicates that the groups are equivalent. Given unequal sample sizes across age and modality, results are reported for Welch's t-test because it does not rely on the assumption of equal variances (Delacre, Lakens, \& Leys, 2017; Lakens, 2017). The goal of the preliminary study was to calibrate tasks in the range of moderately difficult to moderately easy ( $d$ ' value between 1.5 and 2.5 ), with a target $d$ ' value of 2.5; therefore, the lower and upper bounds (raw 
Table 1 Task matching

\begin{tabular}{|c|c|c|c|c|c|}
\hline Age & Modality & Task-matching differences & Linear equation & $\mathrm{d}^{\prime}=2.5$ intercept & Final matched differences \\
\hline \multicolumn{6}{|c|}{ Children } \\
\hline & Visual & $\begin{array}{l}9.6-40 \% \\
0.12-0.5 \text { in. }\end{array}$ & $y=.03 x+1.62$ & $29.3 \%$ & $\begin{array}{l}29.6 \% \\
0.37 \text { in }\end{array}$ \\
\hline & Auditory & $\begin{array}{l}4-10 \% \\
17.6-44 \mathrm{~Hz}\end{array}$ & $y=.55 x-2.00$ & $8.2 \%$ & $\begin{array}{l}8 \% \\
35.2 \mathrm{~Hz}\end{array}$ \\
\hline \multicolumn{6}{|c|}{ Adults } \\
\hline & Visual & $\begin{array}{l}5-25 \% \\
0.06-0.31 \text { in. }\end{array}$ & $y=.12 x+1.18$ & $11.0 \%$ & $\begin{array}{l}11.2 \% \\
0.14 \text { in }\end{array}$ \\
\hline & Auditory & $\begin{array}{l}2-7 \% \\
8.8-30.8 \mathrm{~Hz}\end{array}$ & $y=.16 x+1.81$ & $4.3 \%$ & $\begin{array}{l}4.25 \% \\
18.7 \mathrm{~Hz}\end{array}$ \\
\hline
\end{tabular}

scores) were set to -1 and 1, respectively. An Excel spreadsheet developed by Lakens (2017) was used to perform the TOST procedure for Welch's t-test. The results indicated that the following were equivalent: (1) visual tasks for children and adults, $t(16.97)=-3.05, p=.004$; (2) auditory tasks for children and adults, $t(13.37)=1.85, p=.044$; (3) visual and auditory tasks for children, $t(17.29)=2.14, p=.024$; (4) visual and auditory tasks for adults, $t(9.62)=1.87, p=.048$.

Overall, the results of the preliminary study provide support for the statistical equivalency of the final stimulus values used in the visual and auditory tasks for children and adults at an intermediate rate ( 20 events $/ \mathrm{min})$. These tasks were used in the main study to assess effects of event rate and temporal expectancy on CPT performance of children and adults using the intermediate event rate examined in the preliminary study and faster and slower event rates.

\section{Main study}

The main study was designed to determine whether manipulations of event rate and temporal expectancy in equated visual and auditory tasks would differentially affect performance of children and adults. The first aim was to investigate how performance changes when stimuli, which have been equated across age and modality at an intermediate event rate, are presented at faster or slower rates. Although event rate has been explored in research with children and adults, results

Table 2 Final stimulus values of neutral event and critical signals for children and adults in the visual and auditory tasks

\begin{tabular}{lll}
\hline & Visual & Auditory \\
\hline Neutral event & 1.25 -in. square & $440.0-\mathrm{Hz}$ tone \\
Critical signal - children & 1.62 -in. square & $475.2-\mathrm{Hz}$ tone \\
Critical signal - adults & 1.39 -in. square & $458.7-\mathrm{Hz}$ tone \\
\hline
\end{tabular}

have been conflicting. The main study sought to provide a better understanding of developmental differences in the effects of event rate on continuous performance. A related aim was to consider the possibility that effects of event rate may depend on whether information is presented in the visual or auditory modality. The final aim of the main study was to explore the effects of violations in temporal expectancy by adding temporally unexpected early and late events to temporally regular (certain) visual and auditory tasks.

\section{Method}

\section{Participants}

Forty-one children were recruited from the local elementary schools in Northeast and Northwest Ohio. As in the preliminary study, parents were asked to report any history of attention problems. The children were paid $\$ 15$ for their participation. Sixty-three adults who were undergraduate students at a university in Northwest Ohio participated in this study in return for course credit. Thirty-three children and adults were omitted from the final analyses for exclusionary criteria: diagnoses or concerns related to attention $(n=5)$, incomplete visual or auditory data $(n=7)$, concerns about participant behavior (e.g., falling asleep or answering a phone) during the task $(n=5)$, computer error $(n=1)$, and hearing screening failure $(n=1)$. Research suggests that musicians are better at frequency (pitch) discrimination than non-musicians (e.g., Micheyl, Delhommeau, Perrot, \& Oxenham, 2006; Tervaniemi, Just, Koelsch, Widmann, \& Schröger, 2005). To reduce the effects of musical training in the auditory task, only adults who met non-musician criteria - no more than 3 years of musical training and no musical training in the last 5 years - were included in the final sample ( $n=14$ excluded). ${ }^{2}$ This classification is similar to criteria used in previous studies

\footnotetext{
${ }^{2}$ When musicians were included in the data analysis, the pattern of results was the same; the only notable difference was an overall increase in $d$ ' scores.
} 
to identify non-musicians (Bidelman, Hutka, \& Moreno, 2013; Fuller, Galvin, Maat, Free, \& Başkent, 2014; Liang et al., 2016). The final sample consisted of 33 children, ranging in age from 7 years 1 month to 8 years 11 months, with an average of 8 years 2 months, and 38 adults, ranging in age from 18 to 27 years, with an average of 19 years 2 months.

\section{Procedure}

All participants completed both a visual and an auditory task on separate occasions, at least $24 \mathrm{~h}$ apart. Within each age group, half of the participants were randomly assigned to perform the visual task first, followed by the auditory task, while the remainder of the participants performed the tasks in the reverse order. The stimuli were identical to those used in the preliminary study (see Table 2). Participants completed 10min visual and auditory tasks at one of three event rates: fast (40 events/min), intermediate (20 events/min), or slow (10 events/min).

In each task, $30 \%$ of the stimuli were critical signals, while the remaining $70 \%$ were neutral events. In addition, during the testing sessions, the temporal expectancy of critical signals and neutral events was varied. For $80 \%$ of the critical signals and neutral events there was a fixed inter-stimulus interval based on the tested event rate (i.e., events were on time with the regular rhythm established by the tested event rate). For the remaining $20 \%$ of the trials, however, events occurred $25 \%$ early or $25 \%$ late relative to the established rhythm of the event stream. A breakdown of the events can be seen in Fig. 1 .

\section{Results}

The results are presented in two sections. First, the effects of event rate in the visual and auditory tasks for children and adults were examined for each of the dependent measures: perceptual sensitivity $\left(\mathrm{d}^{\prime}\right)$, response bias $(\mathrm{c})$, reaction time (RT), hits, and false alarms for the on-time events. Hits and

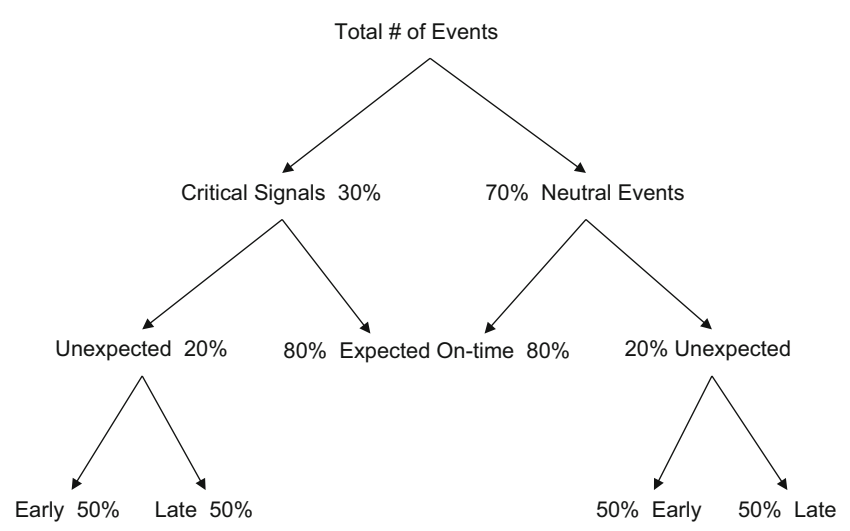

Fig. 1 Diagram of the proportion of early, on-time, and late critical signals and neutral events false alarms were included in the event-rate analyses to allow for comparisons to previous studies that only reported hits and/or false alarms. Second, effects of temporal expectancy were considered for $d$, c c, and RT to determine if participants attended differently to on-time events compared to unexpectedly early and late events. For all analyses, Mauchly's test of the violation of the assumption of sphericity was conducted. Where applicable, the Huynh-Feldt correction was employed.

\section{Event rate}

Separate 2 (age) $\times 3$ (event rate) $\times 2$ (modality) mixed ANOVAs, with repeated measures on modality, were performed on each of the five dependent measures. The means and standard errors for $d^{\prime}$, c, RT, hits, and false alarms for children and adults in the visual and auditory tasks for fast, intermediate, and slow event rates are reported in Table 3.

Perceptual sensitivity ( $d^{\prime}$ ) and response bias (c) The ANOVA on $d^{\prime}$ ' showed no significant main effects of age, event rate, or modality (all $p \mathrm{~s}>0.05$ ), but did reveal a significant two-way interaction between age and modality, $F(1,65)=5.52, p=$ $.02, \eta_{\mathrm{p}}{ }^{2}=.08$ (see Fig. 2). Subsequent pairwise comparisons using Bonferroni-corrected t-tests revealed that adults $(M=$ 2.36) had significantly higher $d$ ' than children $(M=1.58)$ in the auditory task $(p=.04)$. Additionally, children had significantly higher $d^{\prime}$ in the visual $(M=2.27)$ compared to the auditory $(M=1.58)$ task $(p=.02)$. The ANOVA on $\mathrm{c}$ revealed a significant main effect of event rate, $F(2$, $65)=15.48, p<.001, \eta_{\mathrm{p}}^{2}=.32$, with more conservative response bias in the fast $(M=.42)$ compared to the intermediate $(M=.04)$, and slow $(M=-.01)$ conditions (Bonferroni-corrected: $p s<.001$ ). The remaining sources of variance were not significant ( $p s>.05)$.

Reaction time (RT) The ANOVA on RT showed a significant main effect of age, $F(1,65)=11.73, p=.001, \eta_{\mathrm{p}}{ }^{2}=.15$, event rate, $F(2,65)=40.53, p<.001, \eta_{\mathrm{p}}{ }^{2}=.56$ and modality, $F(1$, $65)=85.83, p<.001, \eta_{\mathrm{p}}{ }^{2}=.57$. In general, adults $(M=$ $562.98)$ responded more quickly than children $(M=669.34)$; as event rate slowed, RT also slowed $(M=444.73,615.53$, 788.22 , for the fast, intermediate, and slow event rates, respectively, $p \mathrm{~s}<.001)$, and participant responses were faster to visual $(M=513.29)$ compared to auditory critical signals $(M$ $=719.03)$. The ANOVA also revealed a significant interaction between event rate and modality, $F(2,65)=23.47, p<.001$, $\eta_{\mathrm{p}}{ }^{2}=.42$ (see Fig. 3). Subsequent pairwise comparisons using Bonferroni-corrected t-tests to explore the nature of the interaction indicated that RTs were significantly faster in the visual compared to the auditory task when events were presented at the intermediate ( $M=518.57,712.50$, respectively) and slow $(M=589.14,987.30$, respectively) rates $(p \mathrm{~s}<.001)$. However, when events were presented at the fast event rate, RTs were 
Table 3 Performance measures as a function of age, modality, and event rate

\begin{tabular}{|c|c|c|c|c|c|}
\hline \multirow{2}{*}{$\begin{array}{l}\text { Age } \\
\text { Modality } \\
\text { Event rate }\end{array}$} & \multicolumn{3}{|c|}{ Performance measures } & \multirow[t]{2}{*}{ Mean hits } & \multirow[t]{2}{*}{ Mean fa } \\
\hline & Mean $d^{\prime}$ & Mean c & Mean RT & & \\
\hline \multicolumn{6}{|l|}{ Children } \\
\hline \multicolumn{6}{|l|}{ Visual } \\
\hline Fast & $2.60(0.53)$ & $0.25(0.35)$ & $469.72(67.53)$ & $0.84(0.08)$ & $0.08(0.05)$ \\
\hline Intermediate & $2.18(0.97)$ & $0.12(0.40)$ & $540.32(134.20)$ & $0.80(0.13)$ & $0.16(0.14)$ \\
\hline Slow & $2.05(1.06)$ & $-0.06(0.44)$ & $607.49(153.66)$ & $0.81(0.12)$ & $0.21(0.14)$ \\
\hline \multicolumn{6}{|l|}{ Auditory } \\
\hline Fast & $1.51(1.57)$ & $0.63(0.19)$ & $487.22(64.76)$ & $0.53(0.29)$ & $0.13(0.12)$ \\
\hline Intermediate & $1.75(1.28)$ & $0.12(0.41)$ & $798.92(200.92)$ & $0.68(0.20)$ & $0.18(0.17)$ \\
\hline Slow & $1.48(1.64)$ & $0.02(0.63)$ & $1112.34(262.30)$ & $0.67(0.32)$ & $0.29(0.24)$ \\
\hline \multicolumn{6}{|l|}{ Adults } \\
\hline \multicolumn{6}{|l|}{ Visual } \\
\hline Fast & $1.97(0.51)$ & $0.57(0.55)$ & $394.60(53.04)$ & $0.68(0.14)$ & $0.11(0.12)$ \\
\hline Intermediate & $2.13(0.90)$ & $0.03(0.58)$ & $496.82(104.47)$ & $0.83(0.14)$ & $0.21(0.22)$ \\
\hline Slow & $2.26(0.78)$ & $0.00(0.40)$ & $570.78(127.49)$ & $0.83(0.17)$ & $0.15(0.12)$ \\
\hline \multicolumn{6}{|l|}{ Auditory } \\
\hline Fast & $2.98(1.51)$ & $0.31(0.29)$ & $427.36(94.88)$ & $0.81(0.19)$ & $0.07(0.13)$ \\
\hline Intermediate & $1.88(1.80)$ & $-0.01(0.24)$ & $626.07(150.41)$ & $0.76(0.19)$ & $0.26(0.24)$ \\
\hline Slow & $2.20(1.65)$ & $-0.01(0.34)$ & $862.26(291.84)$ & $0.82(0.15)$ & $0.24(0.23)$ \\
\hline \multicolumn{6}{|l|}{ Mean } \\
\hline \multicolumn{6}{|l|}{ Visual } \\
\hline Fast & 2.29 & 0.41 & 432.16 & 0.76 & 0.10 \\
\hline Intermediate & 2.16 & 0.08 & 518.57 & 0.82 & 0.19 \\
\hline Slow & 2.16 & -0.02 & 589.14 & 0.82 & 0.18 \\
\hline \multicolumn{6}{|l|}{ Auditory } \\
\hline Fast & 2.25 & 0.47 & 457.29 & 0.67 & 0.10 \\
\hline Intermediate & 1.82 & 0.06 & 712.50 & 0.72 & 0.22 \\
\hline Slow & 1.84 & 0.01 & 987.30 & 0.75 & 0.27 \\
\hline
\end{tabular}

similar in the visual $(M=432.16)$ and auditory $(M=457.29)$ tasks $(p>.05)$. There was also a significant interaction between age and modality, $F(1,65)=6.04, p=.02, \eta_{\mathrm{p}}{ }^{2}=.09$

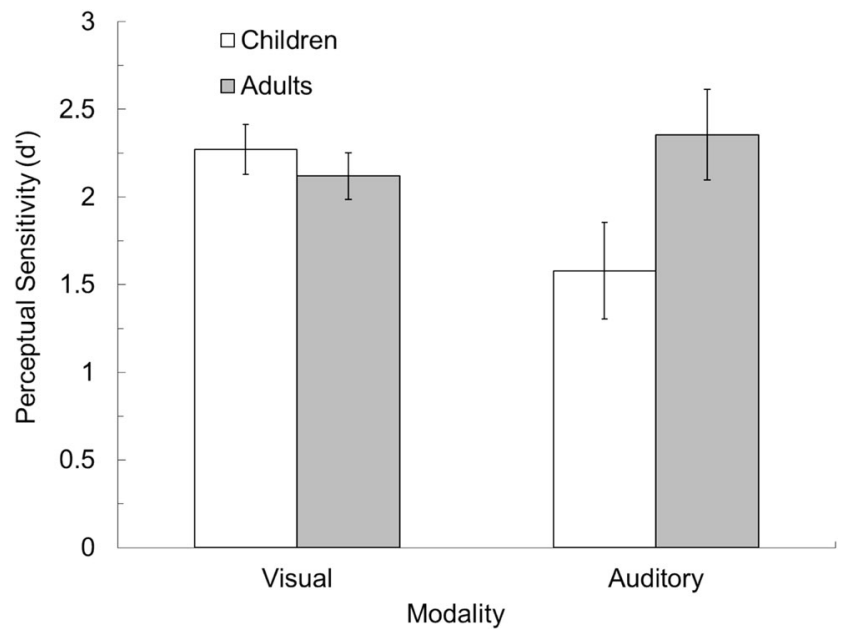

Fig. 2 Mean perceptual sensitivity $\left(d^{\prime}\right)$ with standard error bars as a function of age and modality (see Fig. 4). In exploring the nature of the interaction, subsequent pairwise comparisons using Bonferroni-corrected t-tests revealed that in the auditory task, RTs for children were

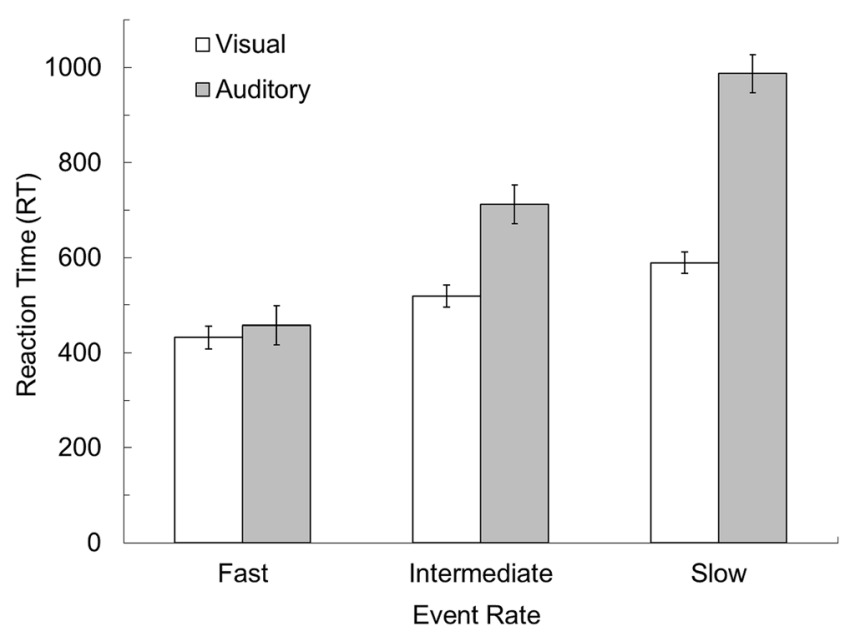

Fig. 3 Mean reaction time (RT) with standard error bars as a function of event rate and modality 


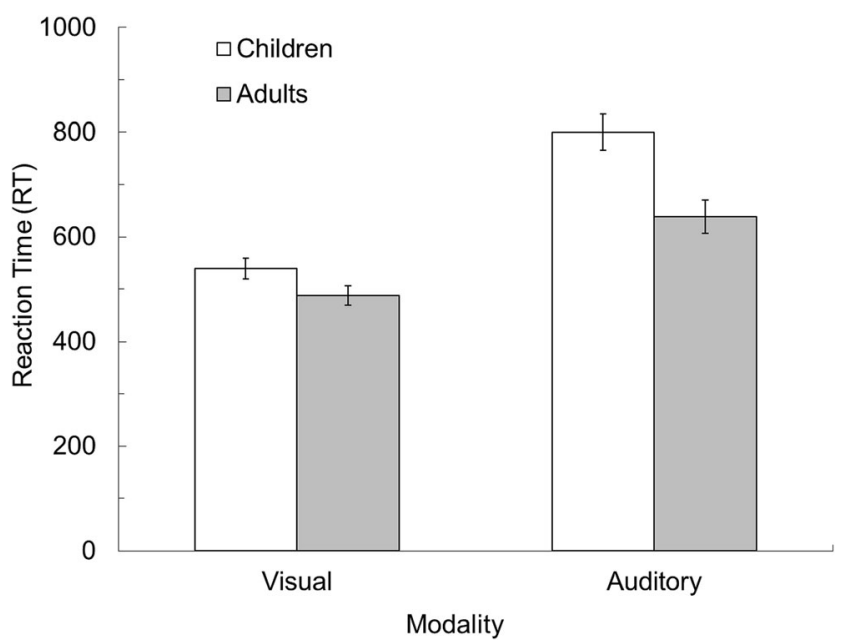

Fig. 4 Mean reaction time (RT) with standard error bars as a function of age and modality

significantly slower $(M=799.50)$ than for adults $(M=$ 638.56), $(p=.001)$; however, in the visual task, RTs were similar for children $(M=539.18)$ and adults $(M=487.40)(p$ $>.05)$. All remaining sources of variance did not reach significance $(p s>.05)$.

Hits and false alarms The ANOVA on hits showed significant main effects of age, $F(1,65)=5.11, p=.03, \eta_{\mathrm{p}}{ }^{2}=.07$ and modality, $F(1,65)=6.68, p=.01, \eta_{\mathrm{p}}{ }^{2}=.09$. In general, adults $(M=0.79)$ correctly detected more critical signals than children $(M=0.72)$ and all participants correctly detected more visual $(M=0.80)$ compared to auditory critical signals $(M=$ 0.71). The ANOVA also revealed a significant two-way interaction between age and modality, $F(1,65)=9.33, p=.003$, $\eta_{\mathrm{p}}{ }^{2}=.13$, and a significant three-way interaction between age, modality, and event rate, $F(2,65)=3.13, p=.05, \eta_{\mathrm{p}}^{2}=.09$. Subsequent pairwise comparisons using Bonferroni-corrected t-tests revealed that the performance of children and adults was similar for the visual and auditory tasks at the intermediate and slow event rates $(p s>.05)$. However, in the fast eventrate condition, there were significant differences in hits for children and adults. Specifically, in the visual task (Fig. 5a), children $(M=.84)$ correctly detected more critical signals than adults $(M=.68)(p=.008)$, while the auditory task (Fig. $5 \mathrm{~b})$ yielded the opposite pattern - adults $(M=.81)$ correctly detected more critical signals than children $(M=.53)(p=.004)$. In the fast event-rate condition, children correctly detected more visual $(M=.84)$ than auditory $(M=.53)$ signals $(p=$ $.001)$. Additionally, in the visual task adults correctly detected fewer visual critical signals at the fast $(M=.68)$ compared to the intermediate $(M=.83)$ and slow $(M=.83)$ event rates $(p=$ .02 and $p=.02$, respectively). However, in the auditory task hits were similar at the fast $(M=.81)$, intermediate $(M=.76)$, and slow $(M=.82)$ event rates $(p>.05)$. All remaining sources of variance did not reach significance ( $p$ s $>.05$ ). The ANOVA on false alarms revealed a significant main effect of event rate,
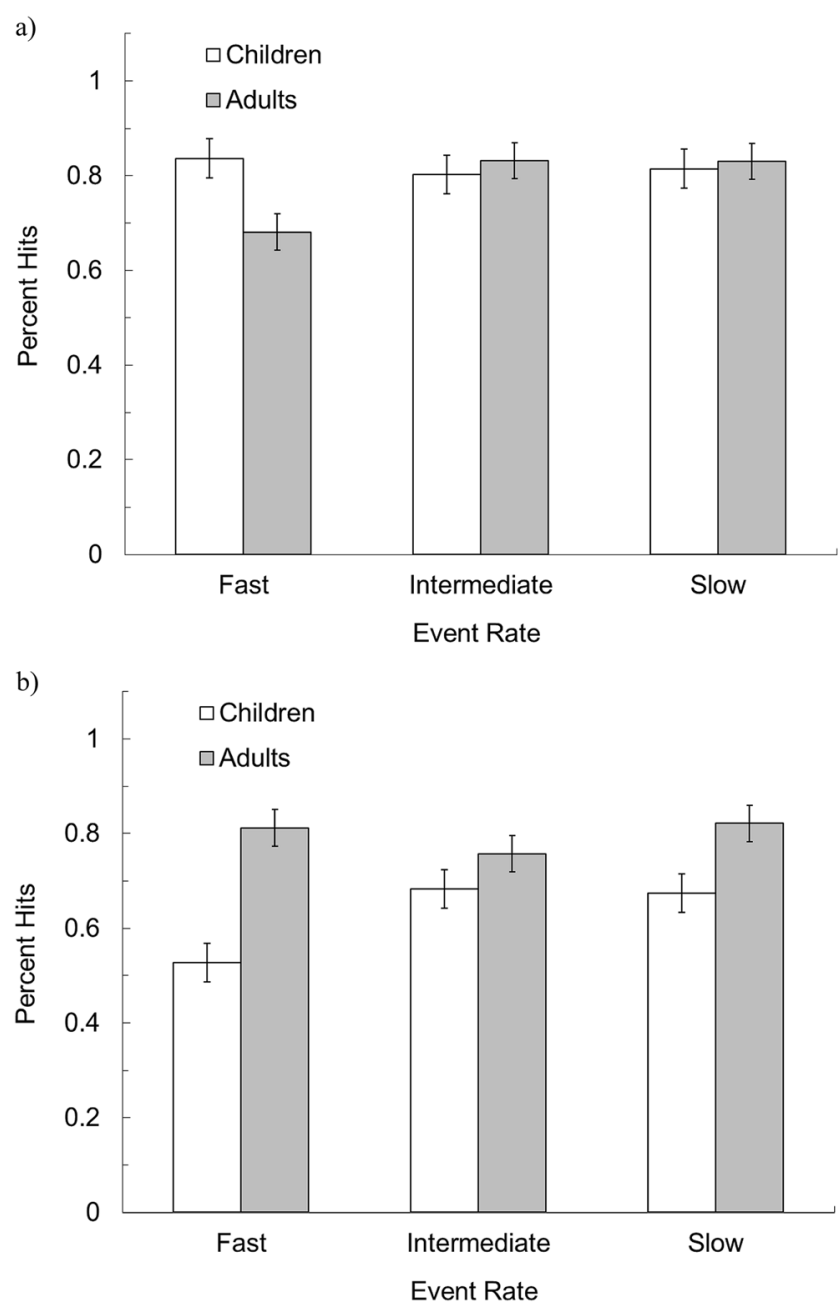

Fig. 5 Mean perceptual sensitivity $\left(d^{\prime}\right)$ with standard error bars as a function of age and event rate for $\mathbf{a}$ visual task and $\mathbf{b}$ auditory task

$F(2,65)=5.29, p=.007, \eta_{\mathrm{p}}{ }^{2}=.14 ;$ participants committed fewer false alarms at the fast $(M=.10)$ compared to the intermediate $(M=.20)$ and slow $(M=.22)$ event rates (Bonferronicorrected: $p=.04$ and $p=.01$, respectively).

\section{Temporal expectancy}

Finally, effects of temporal expectancy, collapsed across event rate, were examined. For this analysis, separate 2 (age) $\times 2$ (modality) $\times 3$ (temporal expectancy) mixed ANOVAs, with repeated measures on the latter two variables, were performed on $d^{\prime}$, c, and RT.

Perceptual sensitivity (d') and response bias (c) The ANOVA on $d^{\prime}$ revealed a significant main effect of temporal expectancy, $F(2,138)=5.24, p=.006, \eta_{\mathrm{p}}^{2}=.07$, with lower $d^{\prime}$ for ontime $(M=2.08)$ compared to early $(M=2.31)$ and late $(M=$ 2.37 ) events (Bonferroni-corrected: $p=.03$ and $p=.01$, respectively). There were no significant effects of temporal expectancy for $\mathrm{c}(p>.05)$. 
Reaction time (RT) The ANOVA on RT yielded no significant main effect of temporal expectancy $(p>.05)$. However, there was a significant interaction between temporal expectancy and modality, $F(1.53,100.84)=3.64, p=.04, \eta_{\mathrm{p}}{ }^{2}=.05$ (see Fig. 6). Subsequent pairwise comparisons using Bonferroni-corrected $\mathrm{t}$-tests revealed that in the visual task, RTs were significantly slower to early $(M=552.12)$ compared to late $(M=495.34)$ events $(p=.006)$. The auditory task produced similar RTs $(M=661.14,697.69,694.82$ for ontime, early, and late events, respectively), regardless of temporal expectancy $(p s>.05)$. The remaining sources of variance failed to reach significance $(p s>.05)$.

\section{Discussion}

In general, the results of the main study revealed differential effects of event rate for children and adults in the visual and auditory tasks. At the fast event rate, children performed better than adults in the visual task, while adults out-performed children in the auditory task. In addition, adult performance in the visual task supports the previously established inverse relationship between performance and event rate (Warm \& Jerison, 1984). However, this relationship was not found in the auditory task. Regarding expectancy, perceptual sensitivity was lowest when events were on time. Although there were no developmental effects, performance was different in the visual and auditory tasks. In the visual task, children and adults responded more slowly when events were early compared to late; however, in the auditory task, performance was similar, regardless of temporal expectancy.

\section{General discussion}

The present study sought to create comparable visual and auditory CPTs, allowing for developmental comparisons

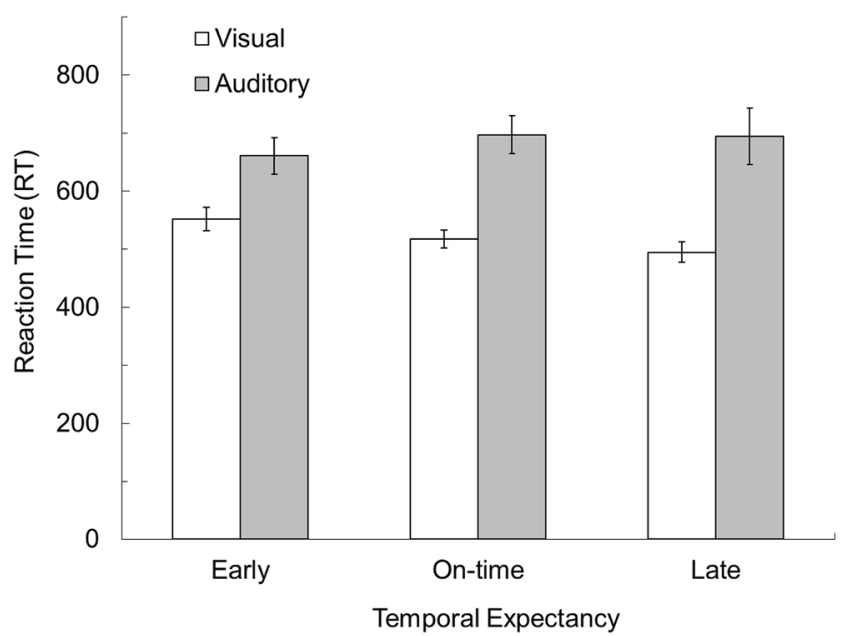

Fig. 6 Mean reaction time (RT) with standard error bars as a function of modality and temporal expectancy concerning the effects of modality, event rate, and expectancy on performance. To achieve that goal, a preliminary study was conducted in which the signal detection index $d$ ' was used to equate tasks across age and modality at an intermediate event rate. Once comparable tasks were established, the main study explored the effects of speeding up and slowing down the task by adding a fast and slow event rate to the previously established intermediate event rate. Further, the study explored how variations in temporal expectancy might differentially affect performance of children and adults in both the visual and auditory CPTs. To assess the influence of temporal expectancy on performance, relatively rare events were introduced that occurred early or late with respect to the regular background rhythm established by the tested event rate.

\section{Matching Tasks across Age and Modality}

The present study provides further support for equating tasks using the perceptual sensitivity index of $d$ '. In the preliminary study, equivalent visual and auditory tasks for children and adults were created. The matching procedure proved efficacious. Although average $d$ ' scores were slightly lower than the target value, two one-sided test (TOST) results indicated that the tasks were equivalent. These findings provide evidence that the tasks are equated across age and modality, and that any changes observed with event rate and expectancy in the main study can be attributed to variable manipulations and not task differences in demands.

\section{Event rate}

In general, developmental differences emerged in the effects of event rate on performance in the visual and auditory CPTs; however, there were overall influences of event rate that were consistent with previous work. Response bias and false alarms were sensitive to the effects of event rate. Considering participants' willingness to respond to events, children and adults were more conservative at the fast event rate than at the intermediate and slow event rates in the visual and auditory tasks. Both children and adults committed the fewest false alarms at the fast event rate, regardless of modality. These results are consistent with effects of event rate on conservatism and false alarms in the visual modality for children and adults (LaurieRose et al., 2005). RT slowed as a function of event rate; participants produced the fastest RTs in the fast event-rate condition and the slowest RTs in the slow event-rate condition. This pattern is consistent with previous findings (Chee et al., 1989; Laurie-Rose et al., 2005); however, it was more pronounced in the auditory compared to the visual task. In addition, the previously reported inverse relationship between performance (i.e., hits) and event rate for adults (Jerison \& Pickett, 1964; Tiwari et al., 2009; Yadav et al., 2016) was observed for adults; however, this effect was only seen in the visual task. 
Performance differences between children and adults were observed in the visual and auditory tasks. When event rate was increased from 20 to 40 events/min in the visual task, developmental differences emerged - children detected more visual critical signals than adults and more visual than auditory critical signals. However, speeding up the auditory task resulted in the opposite pattern - adults detected more auditory critical signals than children. In addition to developmental effects, modality differences at the fast event rate for children were consistent with previous research (Aylward et al., 2002; Borgaro et al., 2003; Curtindale et al., 2007): they detected more critical signals in the visual compared to the auditory task. Similarly, children had greater $d$ ' in the visual compared to the auditory task, regardless of event rate. However, for adults, the fast event rate yielded the opposite pattern - they detected more critical signals in the auditory compared to the visual task. Previous investigations of modality that considered task equivalency and controlled for difficulty produced inconsistent results for adults; either comparable performance for both modalities (See et al., 1995) or better performance (i.e., hits) in the visual compared to the auditory task (Curtindale et al., 2007).

A common explanation for the inverse relationship between performance and event rate is an informationprocessing resource model of attention (Davies \& Parasuraman, 1982; Parasuraman \& Davies, 1977; Parasuraman, Warm, \& Dember, 1987). In general, CPTs require repeated discriminations between critical signals and neutral events that deplete information-processing resources. Fast event rates increase the necessary rate of responding and task demands (Jerison, 1970), draining more of the information-processing resources than slow event rates. Participants may respond to the increased demands of the fast event rate by changing their cognitive strategy; they set a higher criterion for making a response, which results in more conservative responding. In the present study, results for overall response bias are consistent with this model; both children and adults were more conservative when events were presented at the fast compared to the intermediated and slow event rates. Although adults showed the characteristic decrease in correct detections associated with increased event rate, this was only the case for the visual task. The informationprocessing resource model of attention cannot explain adult performance in the auditory task or children's performance in the visual and auditory tasks, beyond the effects of event rate on c. However, these results may be the influenced by differences in task coupling and participant arousal.

Task coupling is the degree to which participants are connected to the task display (Warm \& Jerison, 1984). In a fast event-rate task, if participants do not carefully observe the display at the appropriate time, they may miss critical signals. However, the extent of inappropriate observing can depend on whether the task is loosely or tightly coupled. For example, when observers in a visual task were put in head restraints to make the task more tightly coupled, the effects of event rate decreased (Warm, Wait, \& Loeb, 1976). The proportion of hits in the fast condition increased; however, the level of coupling did not influence performance in the slow eventrate condition. These findings indicate that, at the fast event rate, observing might become easier when tasks are closely coupled (Warm \& Jerison, 1984). According to this argument, an auditory task, which is typically considered more tightly coupled than a visual task, should not yield the same diminished performance seen in visual tasks when event rate is fast. The results of the present study support this explanation. When events were presented in the tightly coupled auditory modality, there were no effects of event rate on hits - adults performed similarly in the fast, intermediate, and slow event-rate conditions. Children, however, did not show the same effects of task coupling. Overall, children had higher $d$ ' scores for the visual compared to the auditory task, and when events were presented at a fast rate, they detected fewer critical signals in the tightly coupled auditory task than in the loosely coupled visual task. These results suggest that task coupling does not have the same impact on children and adults. One factor that may influence the relationship between task coupling and children's performance is arousal.

According to the optimal stimulation theory, an individual's state may be influenced by stimulus presentation rate (Sanders, 1983). For children, who have less experience modulating their arousal and lower levels of arousal than adults, the fast event rate may provide additional stimulation and, thus, improved performance (Zentall \& Zentall, 1983). An arousal-based account of this theory was previously employed by Laurie-Rose et al. (2005) to explain children's superior performance at the fast event rate in a visual task. Similarly, arousal may account for children's fast event rate performance in the present study - they out-performed adults in the visual task because the fast event rate increased their arousal to an optimal level. Children's poorer performance in the auditory compared to the visual task is more complicated. One explanation for this difference is that the tightly coupled auditory task is more stimulating than the visual task because children cannot easily escape the presentation of auditory stimuli (Curtindale et al., 2007). While increased stimulation is not necessarily detrimental to children's performance, when it is combined with the higher resource demands of and increased arousal associated with the fast event rate, children may struggle more in the auditory compared to the visual task. Further research is needed to provide a clearer understanding of how event rate affects auditory attention in adults and the influence of information-processing resources, task coupling, and arousal in children's CPT performance. 


\section{Temporal expectancy}

The present study was the first in the continuous performance literature to explore the effects of temporal expectancy on performance of children and adults within a task by introducing rare events that were early or late relative to the regular rhythm of the task. Overall, perceptual sensitivity was lower for on-time compared to late events. The effects of temporal expectancy on RT were different for the visual and auditory tasks. In the visual task, early events yielded slower reaction times than late events, while reaction times were similar for all events in the auditory task. It is difficult to compare these results with previous investigations of temporal expectancy; those studies explored performance differences between temporally certain (regular) and uncertain (irregular) CPTs (Adams \& Boulter, 1964; Helton et al., 2005; Richter et al., 1981; Scerbo et al., 1987; Scerbo et al., 1986; Warm et al., 1992).

A large body of work examining variations of temporal expectancy within different types of auditory tasks provides evidence of dynamic attending (Jones et al., 2002; McAuley \& Fromboluti, 2014; Penel \& Jones, 2005); participants are able to utilize this anticipatory attending to predict the occurrence of events that are on time. These expectancy effects highlight predictable events rather than events that occur unexpectedly. In addition to future-oriented (anticipatory) attending, individuals can use reactive attending (Penel \& Jones, 2005). Reactive attending occurs when individuals shift their attention toward an unexpected event after it has occurred. This enhancement of unexpected events compared to on-time, predictable events is known as a capture effect (Penel $\&$ Jones, 2005). Accuracy and RT data have been used to determine the presence of expectancy or capture effects (Penel \& Jones, 2005). Expectancy effects produce the highest accuracy for on-time events compared to early and late events. In addition, expectancy effects in studies using speech are reported as faster RTs for on-time (undistorted) vowel sounds compared to early (shortened) and late (lengthened) vowel sounds (Martin, 1979). Conversely, capture effects yield more false alarms and fewer misses (more hits) to early and late events than on-time events. An increase in false alarms and hits for early and late events, which indicates capture effects, is also observed as a decrease in response bias. Additionally, capture effects can be seen in RT differences, with slower RTs to expected events compared to early or late events. The results of the present study indicate the presence of capture effects - higher $d$ ' for early and late events in both tasks and faster RTs for late compared to early events in the visual task rather than expectancy effects.

The differential effects of temporal expectancy on visual and auditory RTs may be the result of coupling and inappropriate observing. As discussed previously, visual and auditory tasks differ in their degree of coupling. In visual tasks, which are considered loosely coupled, participants may miss stimuli when they move their eyes and heads away from the display (Hatfield \& Loeb, 1968). For example, in the present study, participants responded more slowly to early compared to late critical signals in the visual task. It is possible that they became familiar with the rhythm of the events presented during the task ( $80 \%$ on time) and developed expectancies to predict when they needed to attend to the screen to make correct detections. This would allow them to use fewer resources by taking breaks from monitoring the stimuli when they believed events were unlikely to occur; however, they may have been looking away when the unexpected early events occurred. In auditory tasks, which are often presented through headphones, participants may be less likely to miss stimuli (Hatfield \& Soderquist, 1970; Loeb \& Binford, 1971). Therefore, performance may not be as affected by violations in temporal expectancy, as seen in the present study. Although coupling differences influence the effects of temporal expectancy on visual and auditory CPTs, further research is necessary to determine what other factors may also contribute to these differences.

\section{Conclusion}

This study investigated the developmental effects of event rate and temporal expectancy on visual and auditory performance. The preliminary study provided further support for the method of $d$ ' matching as a tool for making developmental comparisons of performance in auditory and visual CPTs. The main study revealed differential effects of the fast event rate for children and adults in the visual and auditory tasks. In addition, the results of the visual task were consistent with the inverse relationship between event rate and performance for adults. However, the same relationship was not found for the auditory task. Regarding expectancy effects, this study manipulated event timing within a task by introducing rare early or late events relative to the regular rhythm of the task. The results were somewhat consistent with previous results and generally supported dynamic attending theory. However, differential effects of temporal expectancy on modality were found. Gaining a better understanding of the effects of sensory modality on attention and temporal expectancy in future research will help identify important developmental differences in visual and auditory attention.

Author note Portions of this research were presented at the 47th Annual Meeting of the Psychonomic Society and the Society for Research in Child Development Biennial Meeting. The author gratefully acknowledges J. Devin McAuley, Cynthia Laurie-Rose, and the members of the Rhythm, Attention, and Perception (RAP) lab at Bowling Green State University for their many helpful suggestions for improvements on earlier versions of the manuscript. 


\section{References}

Adams, J.A., \& Boulter, L.R. (1964). Spatial and temporal uncertainty as determinants of vigilance behavior. Journal of Experimental Psychology, 67, 127.

Aylward, G.P., Brager, P., \& Harper, D.C. (2002). Relations between visual and auditory continuous performance tests in a clinical population: A descriptive study. Developmental Neuropsychology, 21, 285-303.

Baddeley, A.D. \& Colquhoun, W.P. (1969). Signal probability and vigilance: A reappraisal of the "signal-rate" effect. British Journal of Psychology, 60, 169-178.

Ballard, J.C. (1996). Computerized assessment of sustained attention: A review of factors affecting vigilance performance. Journal of Clinical and Experimental Neuropsychology, 18, 843-863.

Bidelman, G.M., Hutka, S., \& Moreno, S. (2013). Tone language speakers and musicians share enhanced perceptual and cognitive abilities for musical pitch: Evidence for bidirectionality between the domains of language and music. PLoS ONE, 8, e60676.

Borgaro, S., Pogge, D.L., DeLuca, V.A., Bilginer, L., Stokes, J., \& Harvey, P.D. (2003). Convergence of different versions of the continuous performance test: Clinical and scientific implications. Journal of Clinical and Experimental Neuropsychology, 25, 283292.Fj

Chee, P., Logan, G., Schachar, R., Lindsay, P., \& Wachsmuth, R. (1989). Effects of event rate and display time on sustained attention in hyperactive, normal, and control children. Journal of Abnormal Child Psychology, 17, 371-391.

Craig, A. (1984). Human engineering: The control of vigilance. In J.S. Warm (Ed.), Sustained attention in human performance (pp. 247 291). Chichester: John Wiley \& Sons.

Curtindale, L.M., Laurie-Rose, C., Bennett-Murphy, L., \& Hull, S. (2007). Sensory modality, temperament, and the development of sustained attention: A vigilance study in children and adults. Developmental Psychology.

Davies, D.R., \& Parasuraman, R. (1982). The psychology of vigilance. London: Academic.

Delacre, M., Lakens, D., \& Leys, C. (2017). Why psychologists should by default use Welch's t-test instead of student's t-test. International Review of Social Psychology, 30.

Fuller, C.D., Galvin, J. J., Maat, B., Free, R.H., \& Bașkent, D. (2014). The musician effect: Does it persist under degraded pitch conditions of cochlear implant simulations?. Frontiers in Neuroscience, 8, 179. doi:https://doi.org/10.3389/fnins.2014.00179.

Green, D.M., \& Swets, J.A. (1966). Signal detection theory and psychophysics (Vol. 1). New York: Wiley.

Hatfield, J.L., \& Loeb, M. (1968). Sense mode and coupling in a vigilance task. Perception \& Psychophysics, 4, 29-36.

Hatfield, J.L., \& Soderquist, D.R. (1970). Coupling effects and performance in vigilance tasks. Human Factors, 12, 351-359.

Helton, W.S., Hollander, T.D., Warm, J.S., Matthews, G., Dember, W.N., Wallaart, M., ... \& Hancock, P.A. (2005). Signal regularity and the mindlessness model of vigilance. British Journal of Psychology, 96 , 249-261.

Jenkins, H.M. (1958). The effect of signal-rate on visual monitoring. American Journal of Psychology, 1, 647-661.

Jerison, H.J. (1970). Vigilance, discrimination, and attention. In D. Mostofsky (Ed.), Attention: Contemporary theory and analysis. New York: Appleton-Century-Crofts, 1970).

Jerison, H.J., \& Pickett, R.M. (1964). Vigilance: The importance of the elicited observing rate. Science, 143, 970-971.

Jones, M.R. \& Boltz, M. (1989). Dynamic attending and responses to time. Psychological Review, 96, 459-491.
Jones, M.R., Moynihan, H., MacKenzie, N., \& Puente, J. (2002). Temporal aspects of stimulus-driven attending in dynamic arrays. Psychological Science, 13, 313-319.

Lakens, D. (2017). Equivalence tests: A practical primer for t tests, correlations, and meta-analyses. Social Psychological and Personality Science, 8, 355-362.

Lasee, M.J., \& Choi, H.S. (2013). Evidence of reliability and validity for a children's auditory continuous performance test. SAGE Open, 3, 2158244013511828

Laurie-Rose, C., Bennett-Murphy, L., Curtindale, L.M., Granger, A.L., Walker, H.B. (2005). Equating tasks and sustaining attention in children and adults: The methodological and theoretical utility of $d$ ' matching. Perception \& Psychophysics, 67, 254-263.

Laurie-Rose, C., Bennett-Murphy, L., Schickedantz, B., \& Tucci, J. (2001). The effects of event rate and signal probability on children's vigilance. Journal of Clinical and Experimental Neuropsychology, $23,215-224$

Laurie-Rose, C., Pempek, T., Curtindale, L. (2015). Sustained attention in infants and children. In Hoffman, R.R., Hancock, P.A., Scerbo, M.W., Parasuraman, R., Szmala, J.L. (Eds.), The Cambridge handbook of applied perception research (Vol. 2, pp. 979-1003). New York, NY: Cambridge University Press.

Liang, C., Earl, B., Thompson, I., Whitaker, K., Cahn, S., Xiang, J., Fu, Q. J., ... Zhang, F. (2016). Musicians are better than non-musicians in frequency change detection: Behavioral and electrophysiological evidence. Frontiers in Neuroscience, 10, 464. doi:https://doi.org/10. 3389/fnins.2016.00464.

Loeb, M., \& Binford, J.R. (1971). Modality, difficulty, and 'coupling' in vigilance behavior. American Journal of Psychology, 84, 529-541.

Macmillan, N.A., \& Creelman, C.D. (1991). Detection theory: a user's guide (Cambridge UP, Cambridge, UK).

Martin, J.G. (1979). Rhythmic and segmental perception are not independent. Journal of the Acoustical Society of America, 65, 1286-1297.

McAuley, J. D., \& Fromboluti, E. K. (2014). Attentional entrainment and perceived event duration. Philosophical Transactions of the Royal Society, B: Biological Sciences, 369, 20130401.

McAuley, J.D., Jones, M.R., Holub, S., Johnston, H.M., \& Miller, N.S. (2006). The time of our lives: Life span development of timing and event tracking. Journal of Experimental Psychology: General, 135, 348.

Metin, B., Roeyers, H., Wiersema, J.R., van der Meere, J., \& SonugaBarke, E. (2012). A meta-analytic study of event rate effects on Go/ No-Go performance in attention-deficit/hyperactivity disorder. Biological Psychiatry, 72, 990-996.

Micheyl, C., Delhommeau, K., Perrot, X., \& Oxenham, A.J. (2006). Influence of musical and psychoacoustical training on pitch discrimination. Hearing Research, 219, 36-47.

Miller, J. E., Carlson, L. A., \& McAuley, J. D. (2013). When what you hear influences when you see: Listening to an auditory rhythm influences the temporal allocation of visual attention. Psychological Science, 24, 11-18.

Parasuraman, R. (1979). Memory load and event rate control sensitivity decrements in sustained attention. Science, 205, 924-927.

Parasuraman, R. \& Davies, D.R. (1977). A taxonomic analysis of vigilance performance. In R. R. Mackie (Ed.), Vigilance: Theory, operational performance, and physiological correlates (pp. 559-574). New York: Plenum Press.

Parasuraman, R., Nestor, P., \& Greenwood, P. (1989). Sustainedattention capacity in young and older adults. Psychology and Aging, 4, 339-345.

Parasuraman, R., Warm, J. S., \& Dember, W.N. (1987). Vigilance: Taxonomy and utility. In L.S. Mark, J.S. Warm, \& R.L. Huston (Eds.) Ergonomics and human factors: Recent research. (pp. 1132) New York: Springer-Verlag. 
Penel, A. \& Jones, M.R. (2005). Speeded detection of a tone embedded in a quasi-isochronous sequence: Effects of a task-irrelevant temporal irregularity. Music Perception, 22, 371-388.

Richter, D.O., Senter, R.J., Warm, J.S. (1981). Effects of the rate and regularity of background events on sustained attention. Bulletin of the Psychonomic Society, 18, 207-210.

Riccio, C.A., Reynolds, C.R., Lowe, P., \& Moore, J.J. (2002). The continuous performance test: a window on the neural substrates for attention?. Archives of clinical neuropsychology, 17, 235-272.

Roebuck, H., Freigang, C., \& Barry, J.G. (2016). Continuous performance tasks: Not just about sustaining attention. Journal of Speech, Language, and Hearing Research, 59, 501-510.

Sanders, A.F. (1983). Towards a model of stress and human performance. Acta Psychologia, 53, 61-97.

Scerbo, M.W., Warm J.S., Doettling, V., Parasuraman, R., \& Fisk, A.D. (1987). Event asynchrony and task demands in sustained attention. In L. S. Mark, J.S. Warm, \& R.L. Huston (Eds.) Ergonomics and human factors: Recent research. (pp. 33-39) New York: SpringerVerlag.

Scerbo, M.W., Warm, J. S., Fisk, A.D. (1986). Event asynchrony and signal regularity in sustained attention. Current Psychological Research and Reviews, 5, 335-343.

Schmidt, S.L., Simões, E.D.N., \& Novais Carvalho, A.L. (2016). Association between auditory and visual continuous performance tests in students with ADHD. Journal of Attention Disorders, 1-6.

Schneider, W., Eschman, A., \& Zuccolotto, A. (2002). E-prime user's guide. Pittsburgh: Psychological Software Tools Inc.

Schuirmann, D.J. (1987). A comparison of the two one-sided tests procedure and the power approach for assessing the equivalence of average bioavailability. Journal of Pharmacokinetics and Biopharmaceutics, 15, 657-680.

See, J.E., Howe, S.R., Warm, J.S., \& Dember, W.N. (1995). Metaanalysis of the sensitivity decrement in vigilance. Psychological Bulletin, 117, 230-249.
Tervaniemi, M., Just, V., Koelsch, S., Widmann, A., \& Schröger, E. (2005). Pitch discrimination accuracy in musicians vs nonmusicians: An event-related potential and behavioral study. Experimental Brain Research, 161, 1-10.

Tiwari, T., Singh, A.L., \& Singh, I.L. (2009). Task demand and workload: Effects on vigilance performance and stress. Journal of the Indian Academy of Applied Psychology, 35, 265-275.

van der Meere, J. \& Stemerdink, N. (1999). The development of state regulation in normal children: An indirect comparison with children with ADHD. Developmental Neuropsychology, 16, 213-225.

Warm, J.S. \& Alluisi, E.A. (1971). Influence of temporal uncertainty and sensory modality of signals on watchkeeping performance. Journal of Experimental Psychology, 87, 303-308.

Warm, J. S., Dember, W. N., Murphy, A. Z., \& Dittmar, M. L. (1992). Sensing and decision-making components of the signal-regularity effect in vigilance performance. Bulletin of the Psychonomic Society, 30(4), 297-300.

Warm, J.S. \& Jerison, H. J. (1984). The psychophysics of vigilance. In J.S. Warm (Ed.), Sustained attention in human performance (pp. 1520). Chichester, U.K.: Wiley.

Warm, J.S., Wait, R.G., \& Loeb, M. (1976). Head restraint enhances visual monitoring performance. Perception \& Psychophysics, 20, 281-286.

Yadav, A.K., Dubey, S., \& Singh, I.L. (2016). Event rate and vigilance: A psychophysiological investigation of mental workload. Journal of the Indian Academy of Applied Psychology, 42, 328.

Zentall, S.S., \& Zentall, T.R. (1983). Optimal stimulation theory: A model of disordered activity and performance in normal and deviant children. Psychological Bulletin, 94, 446-471.

Publisher's note Springer Nature remains neutral with regard to jurisdictional claims in published maps and institutional affiliations. 